\title{
Multi-Objective Robustness Analysis of the Polymer Extrusion Process
}

\author{
Lino Costa ${ }^{1[0000-0003-4772-4404]}$ and António Gaspar-Cunha ${ }^{2[1111-2222-3333-4444]}$ \\ ${ }^{1}$ Department of Production and Systems Engineering, University of Minho, \\ Guimarães, Portugal \\ ${ }^{2}$ Institute of Polymers and Composites, University of Minho, Guimarães, Portugal \\ agc@dep.uminho.pt
}

\begin{abstract}
This paper presents a new approach to robustness analysis in multiobjective optimization problems aimed at obtaining the most robust Pareto front solutions and also at distributing solutions along the most robust regions of the optimal Pareto set. The methodology proposed is applied to a single screw polymer extrusion with the aim to obtain the most robust solutions for the optimization of the extruder geometry. The non-dominated solutions obtained take into account their degree of robustness and the distribution of such solutions along the Pareto front accordingly with specific algorithm parameter values. The results obtained have physical meaning and are in accordance with the practical knowledge about the industrial process studied.
\end{abstract}

Keywords: Polymer Extrusion, Multi-Objective Optimization, Robustness.

\section{Introduction}

It is well known that many practical engineering problems are multi-objective, i.e., they can be described by the existence of various, often conflicting, objectives. A useful way to solve this type of problems consists in using Multi-Objective Evolutionary Algorithms (MOEAs) with the aim of finding the best trade-off between those objectives. These types of algorithms are particularly adequate for solving such category of problems since, instead of a single solution, they use a population of candidate solutions that can be evolved towards the optimal Pareto front, allowing to obtain Pareto frontiers representing the trade-off between the objectives [1].

An important question in the optimization of real problems is the sensitivity of the solutions obtained when small variations of the design variables or of environmental parameters occur. This means that the obtained solutions must be robust, i.e., the performance of the optimal solution(s) should be only slightly affected by changes in those parameters [2]. Usually, the perturbations in the design space or related to environmental parameters are due to unsatisfactory manufacturing accurateness or from noisy design processes, which can be classified into four different categories: a) the performance is affected by noise; b) the value of the design variables change after the optimal solution has been found; c) the process performance is estimated by an ap- 
proximation to a real value; d) and the case where the performance changes with time (dynamic problems). In this work, robustness analysis if the second category will be applied to the optimization of the single screw polymer extrusion [3]:

In multi-objective optimization two type of robustness measures can be used: i) expectation, consisting in replacing the original objective function by a measure of both its performance and expectation in the vicinity of the solution considered; and ii) variance, consisting in adding a further objective that quantifies the deviation in the vicinity of the solution $[4,5]$.

For example, the polymer extrusion process depends on the definition of the machine temperature. In this case, the solutions found for the operating conditions and/or the geometry of the machine cannot be deteriorated in the performance if the environmental temperature changes during the day, which only can be accomplished if robustness of the solutions is considered taking into account the temperature fluctuations.

The objective of this work is to apply the multi-objective robustness approach developed previously by the authors in the optimization of single screw extrusion [6]. For that purpose, initially the robustness methodology proposed will be assessed using a simple case of polymer extrusion process optimization and, then, the methodology will be applied to real industrial situations.

This text is organized as follows: in section 2 the optimization problem will be explained; in section 3 the optimization problem will be explained; section 4 will be devoted to presentation and discussion of the results; and in section 5 the conclusions will be presented.

\section{Robustness in Polymer Extrusion}

\subsection{Extrusion Process}

Figure 1 shows a scheme of the polymer extruder. It is constituted by an Archimedes type screw rotating inside a barrel. The solid polymer in the form of pellets is feed in the hopper were by action of the gravity reaches the interior of the cylinder. Then, by action of the screw rotation the polymer is forced to move forward trough the heated barrel zones were the polymer melts and is created the required pressure to the polymer crosses the die that gave the final shape of the product [6]. The working of the process depends on the polymer properties, operating conditions (screw speed, $N$, and barrel temperature profile, $T_{b}$ ) and of the screw geometry (length screw zones, $L i$, and internal screw diameters, $D i$ ).

The process was modelled numerically using an in-house developed software. The calculations of the relevant performance parameters (output, Q; melt temperature at die exit, T; length of screw required for melting, Length; power consumption, Pot; and mixing degree, WATS). The software is able to calculate these performance objectives as a function of the operating conditions and the screw geometry [6]. 


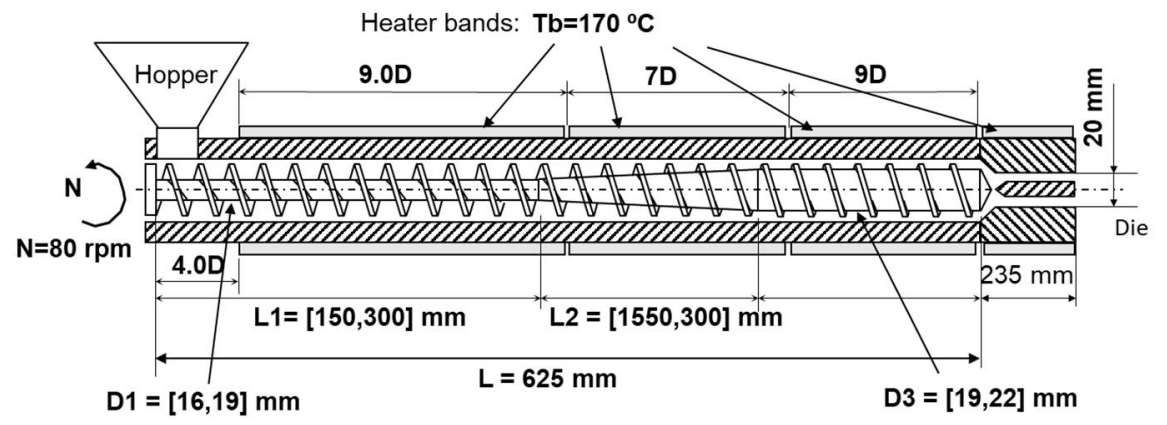

Fig. 1. Single screw extruder decision variables range of variation: operating conditions ( $N$ and $T b)$ and screw geometry $(L 1, L 2, D 1$ and $D 3)$.

\subsection{Robustness Methodology}

The robustness methodology adopted consists in an adaptation of the approach proposed by the authors in Gaspar-Cunha et al. [5] in order to take into account a different MOEA, in this case the SMS-EMOA algorithm [7]. In this algorithm, the fitness of each solution and replacement strategy are based on non-dominated sorting and hypervolume contribution.

In this case robustness of a solution $i$ is computed by the following equation [5]:

$$
R_{i}=\frac{1}{N} \sum_{\substack{j=1 \\ j \neq i}}^{N} \frac{\left|f\left(x_{j}\right)-f\left(x_{i}\right)\right|}{\left|x_{j}-x_{i}\right|}, \quad d_{i j}<d_{\max }
$$

where $N$ is the number of neighbors, $x_{i}$ and $f\left(x_{i}\right)$ are the vectors of decision variables and objectives for solution $i$, respectively, and $d_{i j}$ is the Euclidian distance between vectors $x_{i}$ and $x_{j}$. Only solutions which distances from solution $i$ inferior to $d_{\max }$ contribute for the robustness measure $R_{i}$. All distances were rescaled into the interval $[0,1]$ and $d_{\max }$ was set to 0.1 . If no neighbors are within $d_{\max }(N=0)$ then $R_{i}$ is set to zero.

In order to compute the parameter $\epsilon^{\prime}$ described in Gaspar-Cunha et al. [5] that defines the degree of robustness used to compute the global fitness of solutions, the following parameters values are considered $\gamma=0.2, \delta=0.001$, and $\epsilon=0.1$. These values allow to achieve an adequate balance of non-dominance, diversity, and robustness. Then, the global fitness of a solution $i$ can be computed by

$$
F_{i}=\frac{1}{\operatorname{rank}_{i}}+\left(1-\epsilon^{\prime}\right) \frac{h v_{i}}{h v_{i}+1}+\left(1-\epsilon^{\prime}\right) \frac{R_{i}}{R_{i}+1}
$$

where, for solution $i, \operatorname{rank}_{i}$ is the non-dominance rank, $h v_{i}$ is the contribution of the solution to the global hypervolume of the non-dominated area, and $R_{i}$ is the robustness measure. 


\subsection{Multiobjective Optimization with Robustness}

The multiobjective optimization algorithm with robustness was implemented in MATLAB based on the SMS-EMOA algorithm [7]. The outline of the SMS-EMO is given by Algorithm 1.

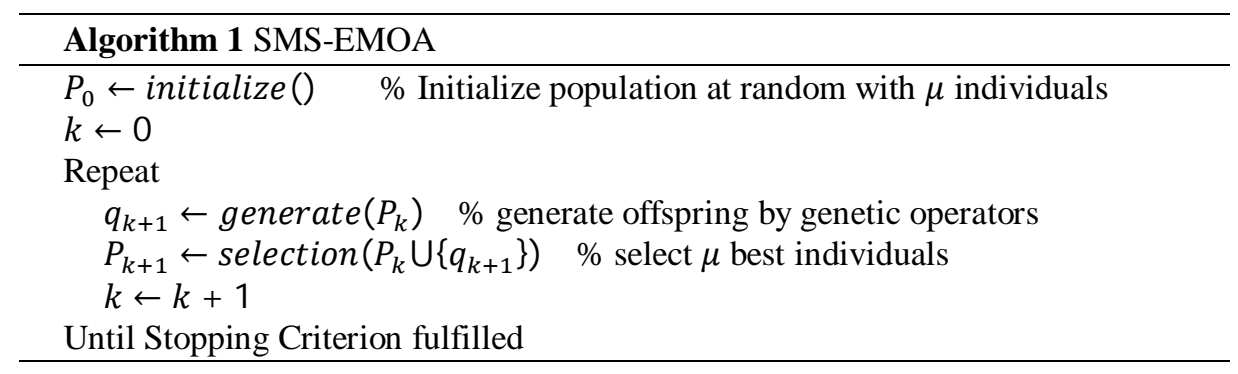

The search starts from an initial population of $\mu$ individuals generated at random satisfying the boundary constraints of the decision variables. In each generation, one single offspring is produced by application of a variation procedure. This procedure can implement genetic operators such as SBX-crossover, polynomial mutation or Gaussian mutation. Next, a deterministic selection procedure selects the $\mu$ best individuals to the next generation. The selection is based on a fitness function that balances three measures (see equation 2): the non-dominated ranking, the hypervolume contribution and the robustness measure.

First, the $P_{k} \cup\left\{q_{k+1}\right\}$ individuals are ranked according to a non-dominated sorting procedure defining $f$ fronts of sets of non-dominated individuals [8]. A rank is assigned to each front representing its level of domination. All solutions belonging to each front are incomparable. The first front $F_{1}$ contains the non-dominated solutions in $P_{k} \cup\left\{q_{k+1}\right\}$. The second front $F_{2}$ contains all non-dominated solutions in $P_{k} \cup\left\{q_{k+1}\right\} \backslash F_{1}$. This procedure is repeated until all solution in $P_{k} \cup\left\{q_{k+1}\right\}$ are included in a front. Any solution in front $F_{i+1}$ is dominated by at least on solution of front $F_{i}$ for $i \geq 1$.

Afterwards, the hypervolume contribution [9] and robustness measure of each individual in $P_{k} \cup\left\{q_{k+1}\right\}$ is computed. Hypervolume definition guaranties that any nondominated solution will not be replaced by a dominated solution since non-dominated solutions will have a higher hypervolume contribution than dominated ones. Hypervolume allows to obtain a well-distributed set of solutions in the objective space as well as to guide the search towards the Pareto optimal front.

For the computation of the robustness measure, a given number of random points are generated in the neighborhood of the solutions in population. If the generated points do not satisfy the boundary constraints of the decision variables, they are projected into the feasible space. These points are then evaluated in terms of the objective function values. Considering all generated points and the solutions in population, equation 1 is used to compute the robustness of solutions. Euclidean distances between decision variables vectors and objective functions vectors are considered in all computations. 
The fitness function expressed by equation 2 balances these three measures. The $\mu$ best individuals in terms of the fitness function are selected to be progenitors of the next generation. This process is repeated until the stopping criterion is fulfilled.

\section{Results and Discussion}

The methodology proposed was tested using a single screw extruder with an internal barrel diameter of $25 \mathrm{~mm}$ and a screw with a length of $625 \mathrm{~mm}$ and processing a High Density Polyethylene. For the purpose of the present study only the maximization of the mass output and the minimization of the length of screw required for melting will be presented.

The objectives of the optimization problem are the output $(\mathrm{kg} / \mathrm{h})$ and the length of screw required for melting $(\mathrm{m})$. This is a bound constrained multi-objective optimization problem with the following decision variables bounds: $150 \mathrm{~mm} \leq L_{1} \leq 300 \mathrm{~mm}$, $150 \mathrm{~mm} \leq L_{2} \leq 350 \mathrm{~mm}, 16 \mathrm{~mm} \leq D_{1} \leq 19 \mathrm{~mm}$, and $19 \mathrm{~mm} \leq D_{3} \leq 23 \mathrm{~mm}$.

The population size was set on 20 individuals. The selection is done using a uniform distribution and variation is performed by the CMA evolution strategy operator [10], which is designed to work with real number representations. The fitness of each solution and replacement strategy are based on the Pareto front rank, the hypervolume measure and robustness measure. For the computation of the robustness measure, four random points are generated in the neighborhood of the solutions in population. The maximum number of generations was set to 20 .

Figure 2 shows the initial and the final populations in the objective space. It can be observed that there is a progress towards the Pareto optimal front during the search, manly for intermediate values of both objectives. Also, the final population follows a linear variation for low (between 3 and $5 \mathrm{~kg} / \mathrm{hr}$ ) and high (between 7 and $8 \mathrm{~kg} / \mathrm{hr}$ ) values of output. The random solutions of the initial population are manly located in the center of the graph, but during the search the algorithm was able to fill the entire space. This can be seen in figure 3 where the size of the circles indicates the number of solutions found on that region.

Therefore, the optimization was accomplished in two different ways: i) finding solutions in the extreme regions and ii) able to find solutions in the intermediate region where the process of extrusion plays and important role. In fact, this behavior observed for intermediate values can be explained by the nature of the problem under consideration.

The observation of figures 4 and 5, where Output and Length were plotted against the decision variables, respectively, allows one to conclude that the solutions found: i) cover almost the entire range of variation allowed for $L_{l}$ (between $150 \mathrm{~mm}$ and $300 \mathrm{~mm}$ ) and $D_{2}$ (between $16 \mathrm{~mm}$ and $19 \mathrm{~mm}$ ); ii) concentrate for the lowest value of $L_{2}$ allowed $(200 \mathrm{~mm})$ and iii) concentrate for the lowest value of $D_{2}(19 \mathrm{~mm})$. In fact, the output is higher wen $D_{2}$ is lower, since in the case the screw channel has its higher value given space to the polymer flows easily in the extruder channel. Also, the gap in the central location is due to the fact that the objective Length (i.e., length required for melting) does not depends linearly from the combination of all decision variables. 
Figure 6 shows the robustness measure as a function of the output. For the solutions of the final population, it can be seen that the less robust solutions are those with larger value of Output. This behavior is due to the slope observed for these solutions in Figure 2 that indicates that a small perturbation in one objective corresponds to a large change in the other. This also means that the extruder process is more sensitive to the decision variables when the aim is to increase the output, which is related with the thermomechanical behavior of the real process.

Figure 7 illustrates the variation of Fitness with Hypervolume. Solutions with larger Hypervolume contributions have a tendency to have a larger Fitness value. Finally, in Figure 8, it is possible to observe that the Fitness of solutions increases with the Robustness measure, as expected.

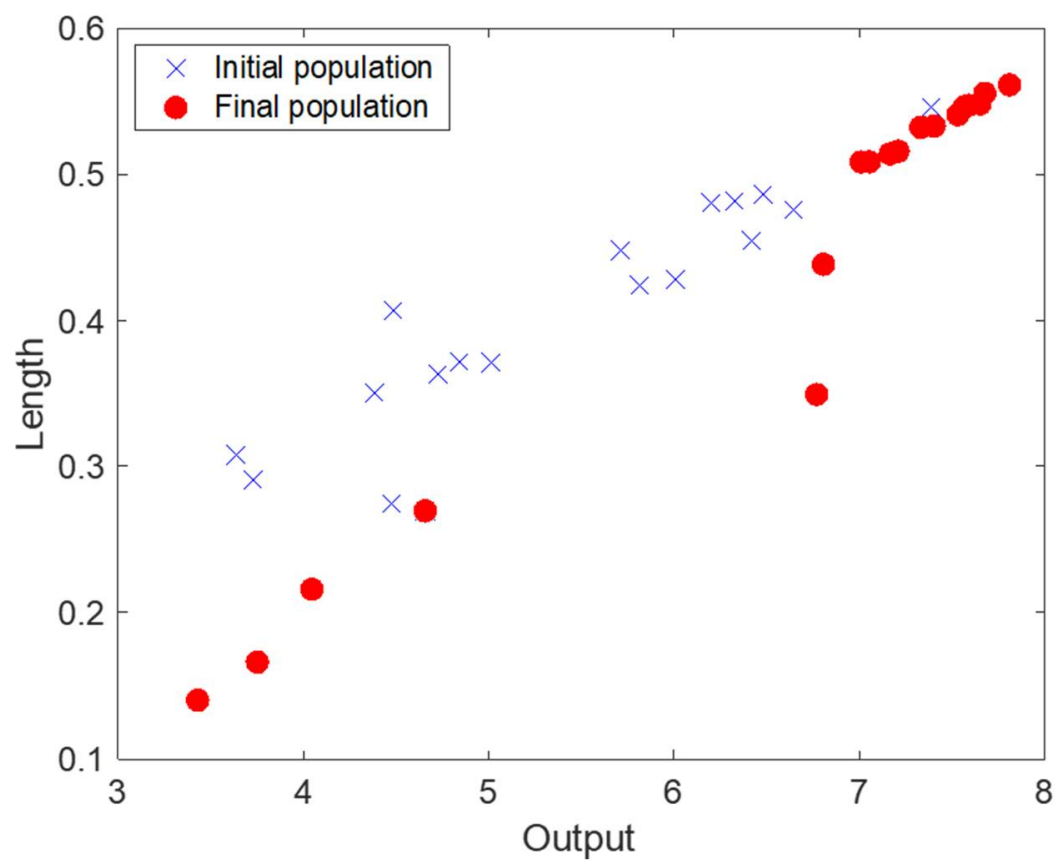

Fig. 2. Initial and final populations: Length (m) versus Output (kg/hr). 


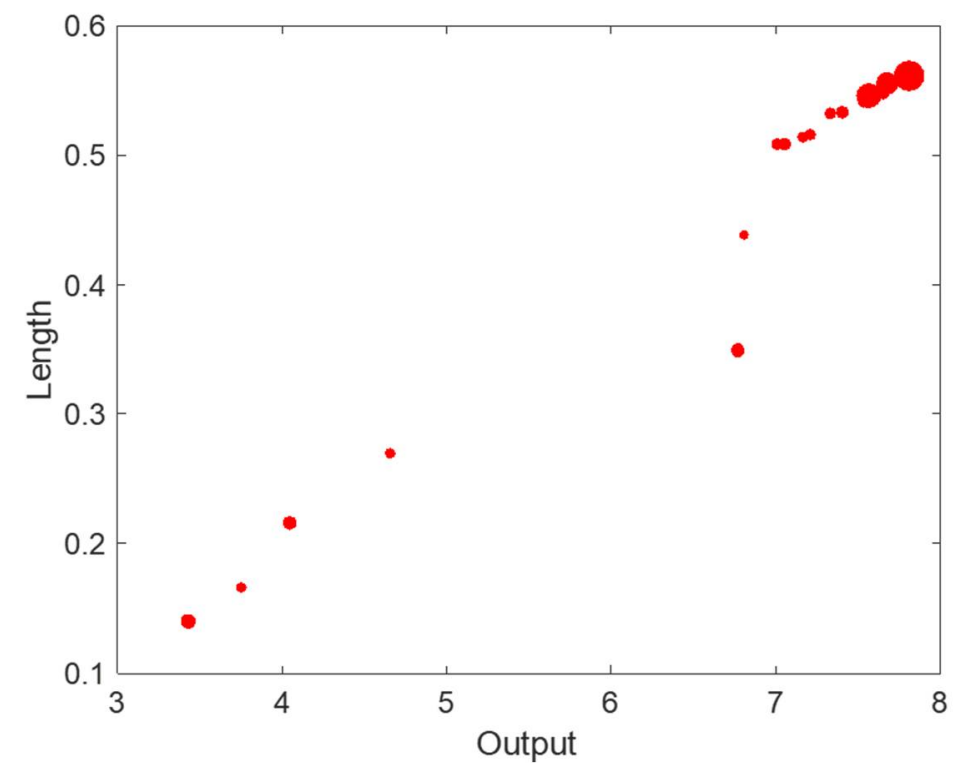

Fig. 3. Quantity of solutions in the final population: Length (m) versus Output (kg/hr).
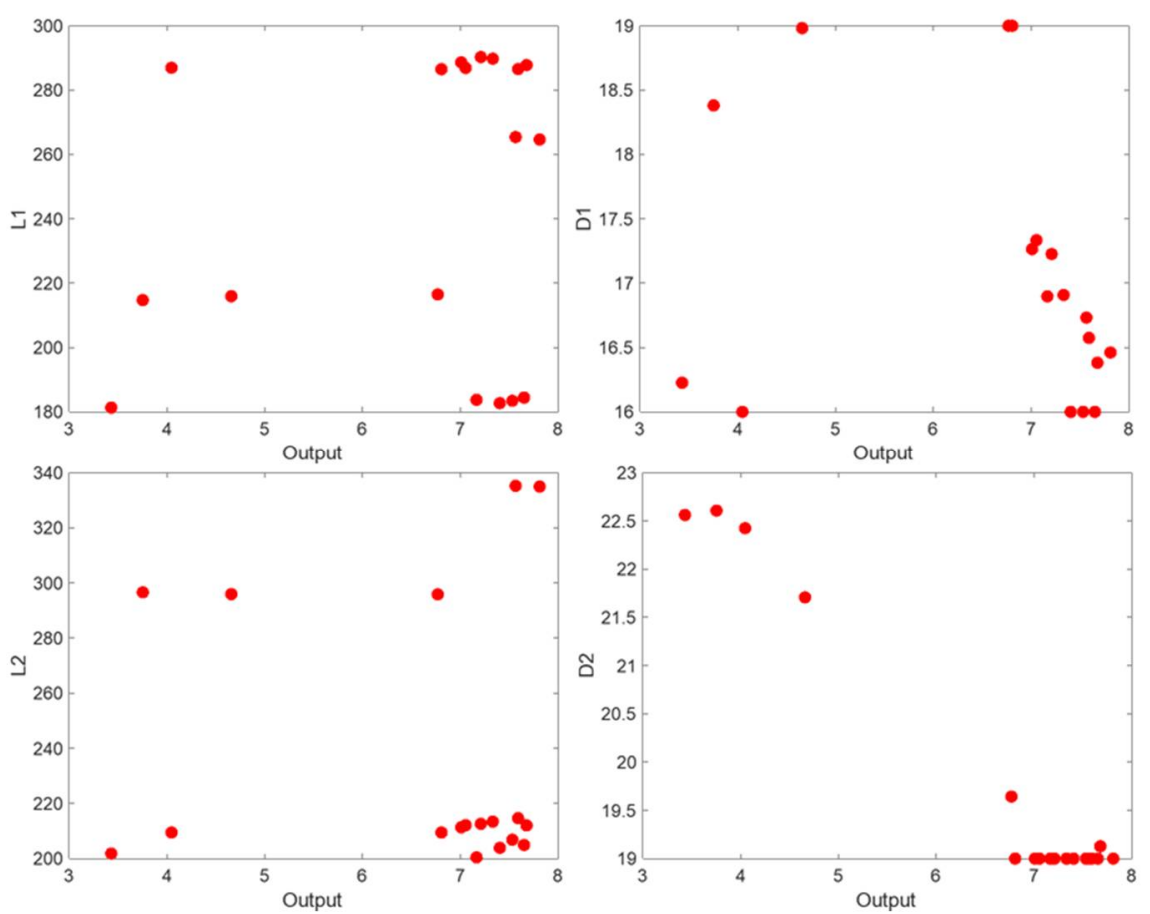

Fig. 4. Final population in the decision space versus Output. 

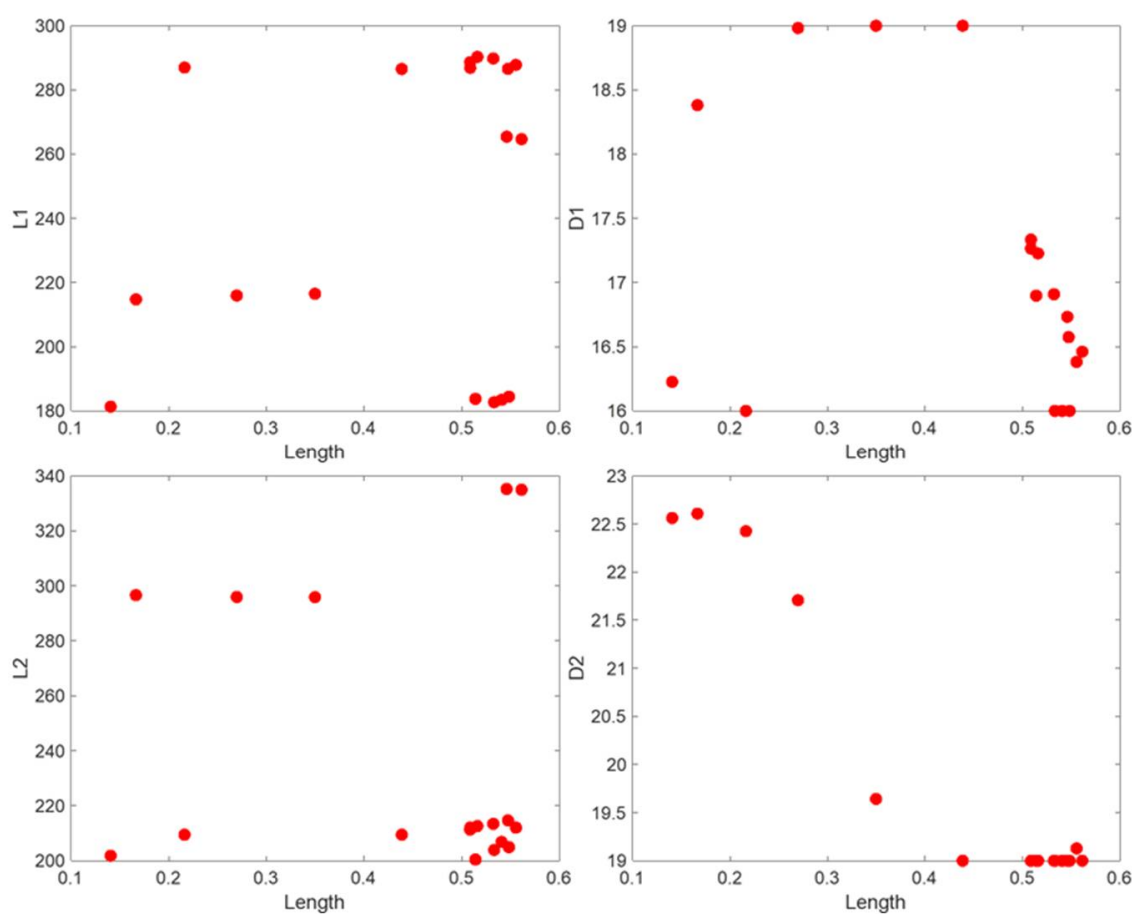

Fig. 5. Final population in the decision space versus Length.

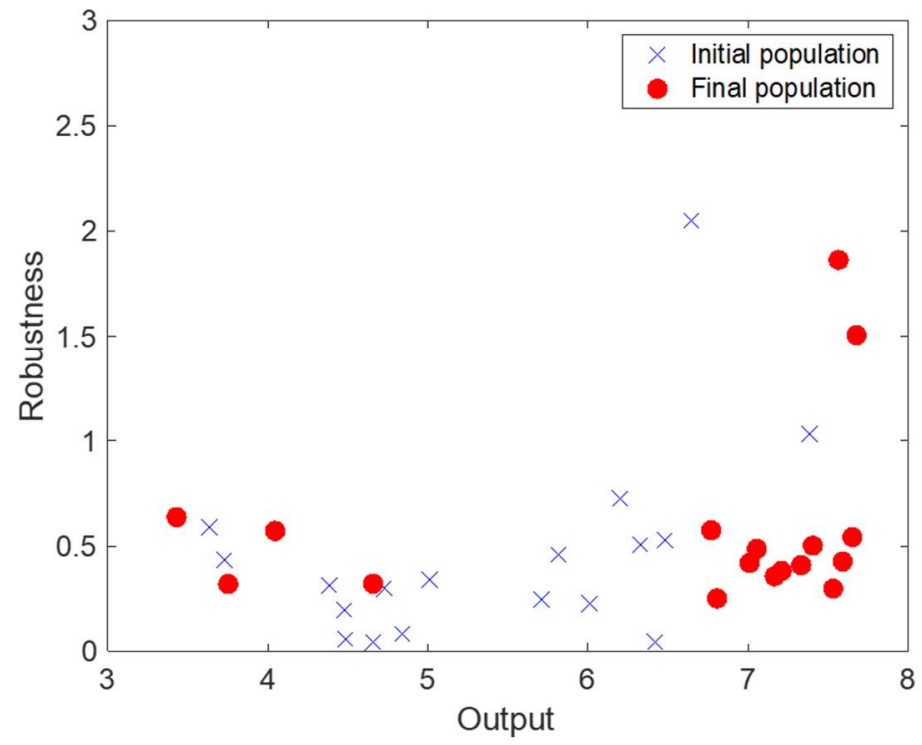

Fig. 6. Initial and final populations: Robustness versus Output (kg/hr). 


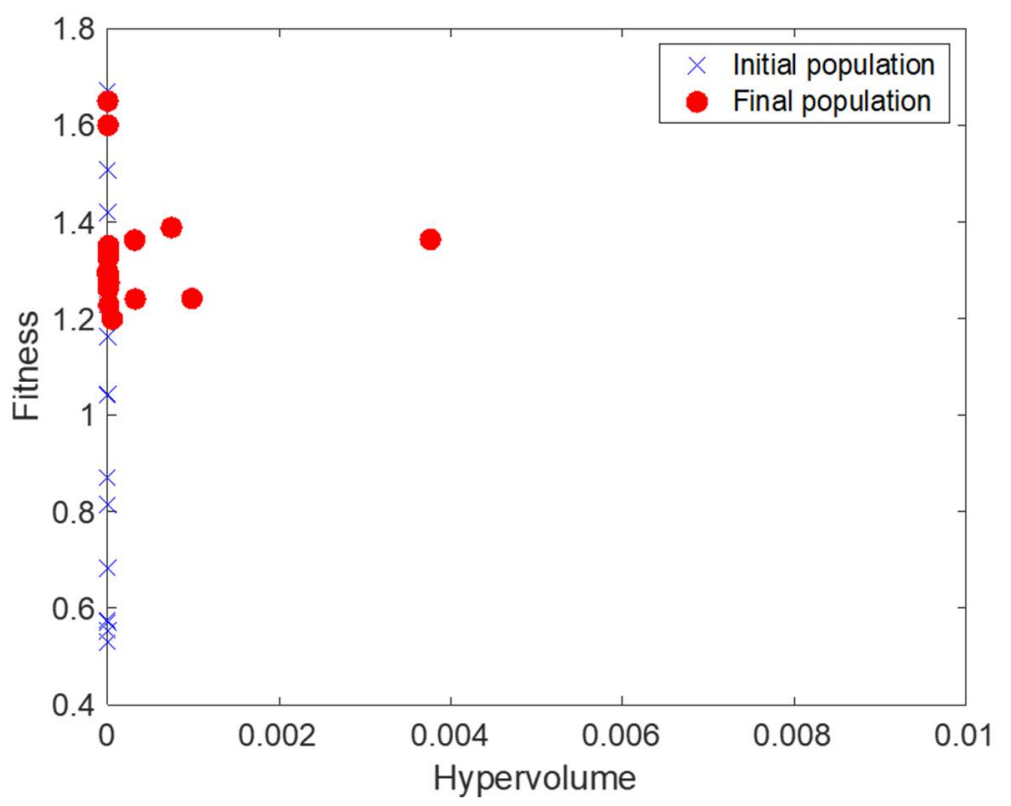

Fig. 7. Fitness versus Hypervolume contributions, initial and final populations.

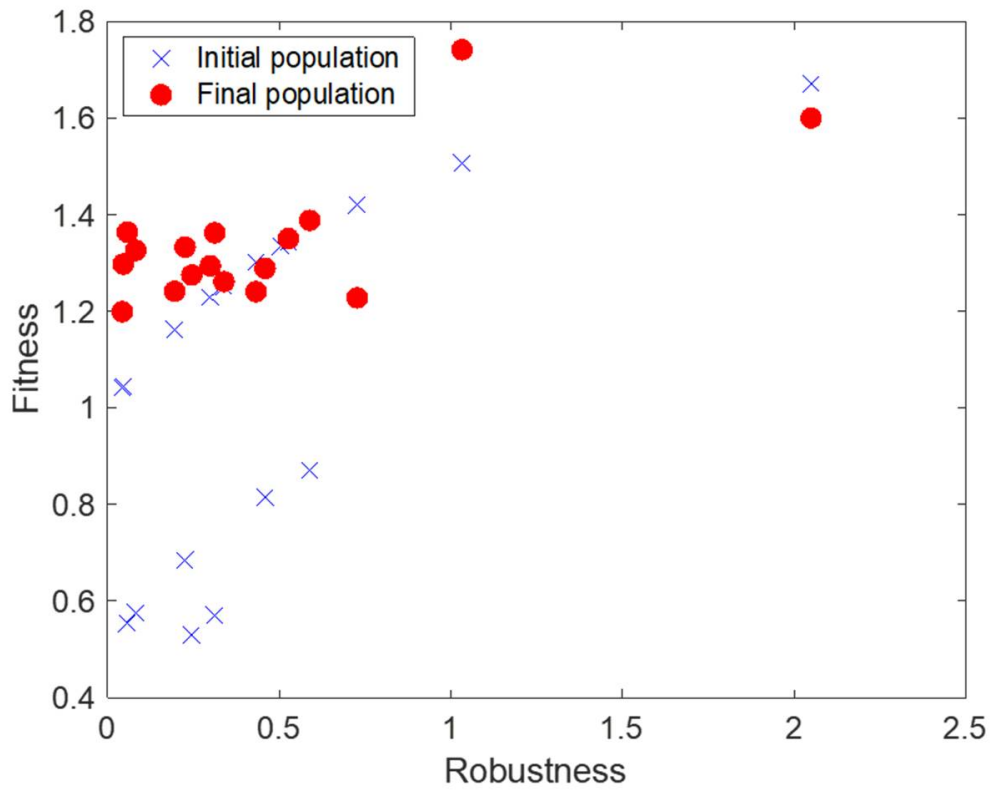

Fig. 8. Fitness versus Robustness of initial and final populations. 


\section{Conclusion}

The results shown allows one to conclude that the solutions obtained have physical meaning in accordance with the knowledge about the polymer extrusion process.

The application of robustness measures together with multi-objective optimization algorithms allow to obtain multiple solutions helping the decision maker in selecting the best solutions considering not only the multi-objective nature of the problem, but also the influence of environmental parameters that are usually uncontrollable.

The application of this methodology to the whole process, i.e., considering all the decision variables and objectives, constitutes a step forward in the engineering of polymer extrusion.

\section{Acknowledgements}

This project has received funding from the European Union's Horizon 2020 research and innovation program under the Marie Skłodowska-Curie grant agreement No 734205 - H2020-MSCA-RISE-2017.

\section{References}

1. Coello, Carlos A., Lamont, G.B. Van Veldhuizen, D.A.: Evolutionary Algorithms for Solving Multi-Objective Problems (Genetic and Evolutionary Computation). SpringerVerlag New York, Inc., Secaucus, NJ, USA (2006).

2. Jin, Y., Olhofer, M., Sendhof, B.: A framework for evolutionary optimization with approximate fitness functions. IEEE Transactions on Evolutionary Computation 6(5), 481-494 (2002).

3. Yaochu, J., Branke, J.: Evolutionary optimization in uncertain environments-a survey. IEEE Transactions on Evolutionary Computation 9(3), 303-317 (2005).

4. Gaspar-Cunha, A., Covas, J.: Robustness in multi-objective optimization using evolutionary algorithms. Computational Optimization and Applications 39(1), 75-96 (2008).

5. Gaspar-Cunha, A., Ferreira, J., Recio, G.: Evolutionary Robustness Analysis for MultiObjective Optimisation: Benchmark Problems. Structural Multidisciplinar Optimization 49(5), 771-793 (2014).

6. Gaspar-Cunha, A., Covas, J.A.: RPSGAe - Reduced Pareto Set Genetic Algorithm: Application to Polymer Extrusion. In: Metaheuristics for Multiobjective Optimisation, Gandibleux, X., Sevaux, M., Sörensen, K., T'kindt, V. (eds.), pp. 221-249, Springer, Berlin, Heidelberg (2004).

7. Beume, N., Naujoks, B., Emmerich, M.: SMS-EMOA: Multiobjective selection based on dominated hypervolume. European Journal of Operational Research 181, 1653-1669 (2007).

8. Deb, K., Pratap, A., Agarwal, S., Meyarivan, T.: A fast and elitist multiobjective genetic algorithm: NSGA-II, IEEE Transactions on Evolutionary Computation 6(2), 182-197 (2002).

9. Emmerich, M., Beume, N., Naujoks, B.: An EMO Algorithm Using the Hypervolume Measure as Selection Criterion, Evolutionary Multi-Criterion Optimization (EMO 2005), LNCS, vol. 3410, pp. 62-76, Springer, Berlin (2005). 
10. Hansen, N.: The CMA Evolution Strategy: A Comparing Review. In J.A. Lozano, P. Larrañaga, I. Inza and E. Bengoetxea (Eds.). Towards a new evolutionary computation. Advances in estimation of distribution algorithms, pp. 75-102, Springer, (2006). 